\title{
Inter-observer agreement according to malaria parasite density
}

\author{
Mounkaila Abdou Billo ${ }^{1 *}$, Mahamadou Diakité2 ${ }^{2}$ Amagana Dolo², Mouctar Diallo², Belco Poudiougou², \\ Sory Ibrahima Diawara ${ }^{2}$, Eric S Johnson ${ }^{1}$, Janet C Rice ${ }^{1}$, Donald J Krogstad ${ }^{1}$ and Ogobara K Doumbo ${ }^{2 *}$
}

\begin{abstract}
Background: Recent developments in diagnostic techniques for malaria, particularly DNA probes and seroimmunology, have raised questions as to how these techniques might be used to facilitate malaria diagnosis at the most peripheral levels of the primary health care system. At present, malaria diagnosis is based on the standard microscopic examination of blood films in most field epidemiologic studies and is likely to remain so in the immediate future in Africa. The objective of this study was to assess inter-observer agreement for the examination of Giemsa-stained slides for Plasmodium falciparum parasites.
\end{abstract}

Methods: Children aged 0 to 10 years were enrolled yearly in Bancoumana village (West Africa), mainly during the transmission season (June to October). The blood smears obtained from the persistently negative children in June 1996, August 1996, October 1996 and March 1997 were systematically re-examined. A stratified random sample (10\%) proportional to the following parasite density classes 1-100, 101-5000, and 5001 and over was taken from the slides collected. The kappa statistics and the intra-class correlation were used as measures of agreement the first and the second slide examinations.

Results: The weighted kappa statistic, widely used as a chance-corrected measure for nominal agreement, showed excellent inter-observer agreement $\left(\mathrm{K}_{\mathrm{w}}=0.7926 ; 95 \% \mathrm{Cl}[0.7588,0.8263] ; p=0.01\right)$. The intra-class correlation co-efficient had the same value of 0.7926 confirming the appropriateness of the weighted kappa statistic. Interobserver agreement for slides read as negative by one observer, or as containing more than 100 parasites per $\mu$ l, was excellent: 97\% (493/506) and 92\% (145/158), respectively. In contrast, the inter-observer agreement for slides read by one observer as containing 1-100 parasites/ $\mu$ l was poor, 36\% (96/268).

Conclusions: In field conditions in Mali, there was a high reproducibility for slides reported as negative or as having more than 100 parasites per $\mu$ l. However, smears with readings of 1-100 parasites per $\mu \mathrm{l}$ were less reproducible and should be re-examined carefully.

Keywords: Inter-observer agreement, Intra-class correlation, Kappa statistic, Parasitaemia, Thick smears, Microscopy

\section{Background}

Measurement error is one of the major sources of bias in epidemiological studies. It can lead to spurious conclusions about the relationship between exposure and disease [1]. Recent developments in diagnostic techniques for malaria, particularly DNA probes and sero-

\footnotetext{
* Correspondence: mounkaila_abdou@hotmail.com; okd@icermali.org ${ }^{1} 1512$ Potomac Ave SE, Washington DC, USA

${ }^{2}$ Mali-Tulane Tropical Medicine Research Center, Malaria Research and Training Center, Faculty of Medicine, Pharmacy and Odontostomatology, University of Sciences, Techniques and Technology, P.O. Box 1805, Bamako, Mali
}

\section{Biomed Central}

immunology, have raised questions as to how these techniques might be used to facilitate malaria diagnosis at the most peripheral levels of the primary health care system [2]. At present, malaria diagnosis is based on the standard microscopic examination of blood film in most field epidemiologic studies and is likely to remain so in the immediate future.

Blood film examinations are crucial not only to distinguish parasitaemic from aparasitaemic children, but also to determine the parasite species and their density in the bloodstream. Thus, a correct reading will reduce misclassification bias and yield accurate effect measures. 
The objective of this study was to assess the reproducibility of the results of thick blood smears obtained from a cohort of children of the village of Bancoumana, Mali (West Africa) by re-examining $~ 10 \%$ of the slides.

\section{Methods}

The blood films were collected and prepared with the approval of both the Research Ethics Committees of the Faculty of Medicine, Pharmacy and Odontostomatology of the University of Bamako, Mali and Tulane University, New Orleans, USA. Study participants were enrolled yearly in Bancoumana village, mainly during the transmission season (June to October). All children aged up to ten years were included in the study. The malaria research and training centre has maintained a field laboratory in Bancoumana since June 1993. This village is located within a narrow riverine valley that has an area of approximately $10 \mathrm{sq} \mathrm{km}$. The village itself consists of approximately 10,000 individuals living in 200 houses. Malaria occurs throughout the year with an average monthly prevalence of approximately $50 \%$, with an intense seasonal transmission from June to November [3].

Thick smears were stained with 3\% Giemsa (Sigma, St Louis, MO, USA) in phosphate buffer ( $\mathrm{pH} 7.0)$ and examined using oil immersion magnification (1,000 X).

The blood smears obtained from the persistently negative children in June, August and October 1996 and March 1997 were systematically re-examined. Also, all negative slides during the four consecutive crosssectional surveys (June 1997 to February 1998) were reexamined. In addition, a stratified random sample (10\%) proportional to the following parasite density classes $1-100,101-5,000$, and 5001 and over was taken from the slides collected in June, August and October 1996 (see Table 1). Each slide was examined under oilimmersion $(100 \times)$ until the microscopist had counted the number of asexual parasites (trophozoites) in fields containing 300 or more white blood cells. Parasite counts were estimated by multiplying the number of asexual parasites per 300 white cells by 25 (based on an average white blood cell count of 7,500 per $\mu \mathrm{l}$ ) and expressed as the number of parasites per $\mu$ l. At least
1,000 white blood cells were counted before a slide was recorded as negative. Slides were then re-examined by an experienced microscopist, blinded to the results of the first readings. Blood smears from the persistently negative children and those negative during the four cross-sectional surveys (June 1997 to February 1998) were re-examined. Of 7,550 thick blood smears, 932 $(12.34 \%)$ were re-examined and classified by parasite density as follows: $0,1-100$ and $>100$ parasites/ $\mu$ l.

The kappa statistics $[4,5]$ and the intra-class correlation were used as measures of agreement the first and the second slide examinations [6]. In addition, Lin's concordance correlation co-efficient for agreement [5] and the limits-of-agreement statistics and graphic procedures $[6,7]$ complemented the aforementioned statistical measures of intra method reliability. The preliminary results showed that agreement is low among positive slides $\leq 100$ per $\mu \mathrm{l}(32.2 \%)$ and very high among negative slides (97.4\%). Therefore, only positive slides with a parasite density $\leq 100$ per $\mu$ l were systematically sampled during the remaining six cross-sectional surveys (March 1997 to February 1998).

A total of 932 slides out of 7,550 (12.34\%) obtained from children of the nested case-control were reread, and 117 out of 1,049 (11.15\%) slides sampled were not seen. Slides were re-read by an experienced microscopist, blinded to the results of the first readings. When the parasite density was $\leq 3$ per 300 leukocytes, a second experienced microscopist re-examined the slide and an average count was reported.

\section{Results}

Out of 7,550, 932 (12.34\%) thick blood slides were re-examined by two well-trained microscopists to measure the reproducibility of the parasite density counts obtained during the cross-sectional surveys (Table 2).

When the measure of interest in a reliability study is an ordered categorical variable, such as the classification of Plasmodium falciparum density in this study, the weighted $\kappa\left(\kappa_{\mathrm{w}}\right)$ is the appropriate measure [4]. The $\kappa_{\mathrm{w}}$ was calculated for the data presented in Table 2 . The $\kappa_{\mathrm{w}}$ shows high agreement, with a result of $0.7926(p<0.00001 ; 95 \%$ CI [0.7588,

Table 1 Numbers of slides sampled and re-examined by parasite count category from June 1996 to March 1998

\begin{tabular}{|c|c|c|c|c|c|c|c|c|c|c|c|c|c|c|c|c|c|c|}
\hline \multirow[b]{2}{*}{ Parasitaemia } & \multicolumn{2}{|c|}{ June 96} & \multicolumn{2}{|c|}{ Aug. 96} & \multicolumn{2}{|c|}{ Oct. 96} & \multicolumn{2}{|c|}{ March 97} & \multicolumn{2}{|c|}{ June 97} & \multicolumn{2}{|c|}{ Aug. 97} & \multicolumn{2}{|c|}{ Oct. 97} & \multicolumn{2}{|c|}{ Feb. 98} & \multicolumn{2}{|c|}{ March 98} \\
\hline & $S^{*}$ & $E^{* *}$ & $S$ & $E$ & $S$ & $E$ & $S$ & $E$ & $S$ & $E$ & $S$ & $E$ & $S$ & $E$ & $S$ & $E$ & $S$ & $E$ \\
\hline 0 & 136 & 120 & 136 & 110 & 136 & 120 & 134 & 112 & 20 & 20 & 20 & 19 & 20 & 20 & 20 & 19 & 0 & 0 \\
\hline $1-100$ & 54 & 48 & 32 & 27 & 22 & 13 & 34 & 29 & 35 & 35 & 29 & 28 & 22 & 20 & 26 & 26 & 35 & 35 \\
\hline $101-5,000$ & 40 & 39 & 40 & 37 & 40 & 37 & 0 & 0 & 0 & 0 & 0 & 0 & 0 & 0 & 0 & 0 & 0 & 0 \\
\hline $5,001+$ & 6 & 6 & 6 & 6 & 6 & 6 & 0 & 0 & 0 & 0 & 0 & 0 & 0 & 0 & 0 & 0 & 0 & 0 \\
\hline Total & 236 & 213 & 214 & 180 & 204 & 176 & 170 & 141 & 55 & 55 & 49 & 47 & 42 & 40 & 46 & 45 & 35 & 35 \\
\hline
\end{tabular}

*Sampled.

**Located and re-examined. 
Table 2 Distribution of Plasmodium falciparum counts by readings

\begin{tabular}{llllll}
\hline \multicolumn{7}{c}{ Second readings } \\
\hline First readings & & $\mathbf{0}$ & $\mathbf{1 - 1 0 0}$ & $\geq \mathbf{1 0 1}$ & Total \\
& $\mathbf{0}$ & 493 & 1 & 12 & 506 \\
& $\mathbf{1 - 1 0 0}$ & 110 & 96 & 62 & 268 \\
& $\geq \mathbf{1 0 1}$ & 5 & 8 & 145 & 158 \\
& Total & 608 & 105 & 219 & 932 \\
\hline
\end{tabular}

0.8263]). The intra-class correlation co-efficient had the same value of 0.7926 , confirming the appropriateness of the weighted kappa statistics [8]. The observed agreement among the negative slides was excellent, 97.43\% (493/506). Conversely, the observed agreement for positive $\leq 100$ was poor, 35.82\% (96/268).

Collapsing the data in a $2 \times 2$ table according to the presence or absence of $P$. falciparum parasite in a thick blood smear gives a Cohen's $\mathrm{k}$ of 0.7179 ( $p<0.0001 ; 95 \%$ CI $[0.6737,0.7621])$, indicating a excellent reproducibility between the first and the second readings.

Figure 1 shows the concordance correlation coefficient [5] computed on the logarithm transformation of the parasite density using William's method [lnpf = $(\mathrm{pf}+1)$ ]. This correlation co-efficient [5] was $0.835,95 \%$ CI (0.816-0.855) using logarithm-transformed parasite counts, and yielded a regression line with near-perfect concordance between the first and the second readings: an average difference of $-0.088 \pm 0.474$ [9] (Figure 2).

\section{Discussion}

Currently, in many African countries, the accepted diagnostic technique for malaria is the examination of stained blood films under the oil immersion lens of the microscope. Serology and molecular technique play a part in epidemiology and in various special investigations $[10,11]$. Light microscopy has a central role in parasite identification and quantification and remains the main method of parasite-based diagnosis in clinic and hospital settings. Thick blood films allow a rapid examination of a relatively large volume of blood, enabling the detection of even scanty parasitaemia of all blood parasites. A wellprepared thick blood film gives more than a ten-fold increase in sensitivity over thin films [12].

Malaria prevalence is decreasing in many African countries. Therefore, the ability to identify all parasites becomes increasingly important. Good quality microscopy conducted by skilled technicians with capacity to manage appropriate quality control, and the currently available rapid diagnosis test (RDT), requiring less training than microscopy, are generally adequate for diagnosis in people who have acute malaria [12]. However, there are issues to be addressed with both procedures. Ensuring the quality of microscopy used for routine diagnosis has often proved difficult as the sensitivity and specificity of routine microscopy is significantly lower when compared with that of qualified microscopists based in reference laboratories [13]. This underlies the need for good training in microscopy for staff in remote areas. The choice of routine diagnosis of malaria in areas of low parasitaemia is microscopy, which is technically more difficult but is better for species identification and for estimating parasite densities, or diagnosis with the user-friendly RDT, which gives a positive or negative result (but not a measure of the density of parasites) and is not good for detecting Plasmodium vivax and the other non-falciparum parasites.

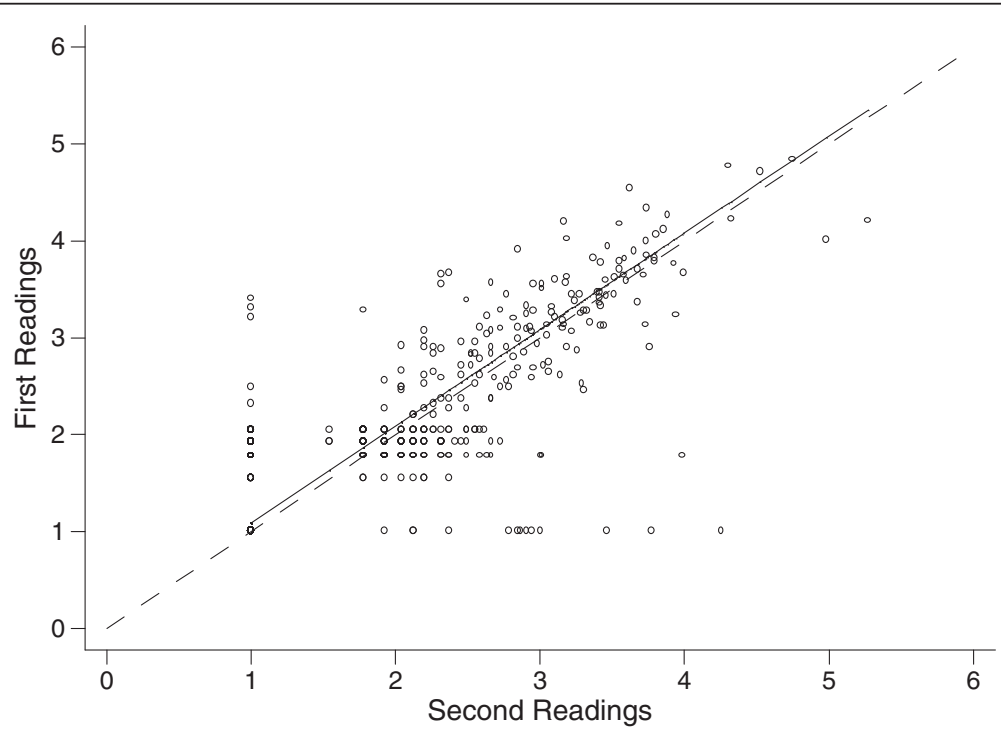

Figure 1 Transformed parasite counts of first and second readings, with line of perfect concordance and regression line using all data (932 slides). 


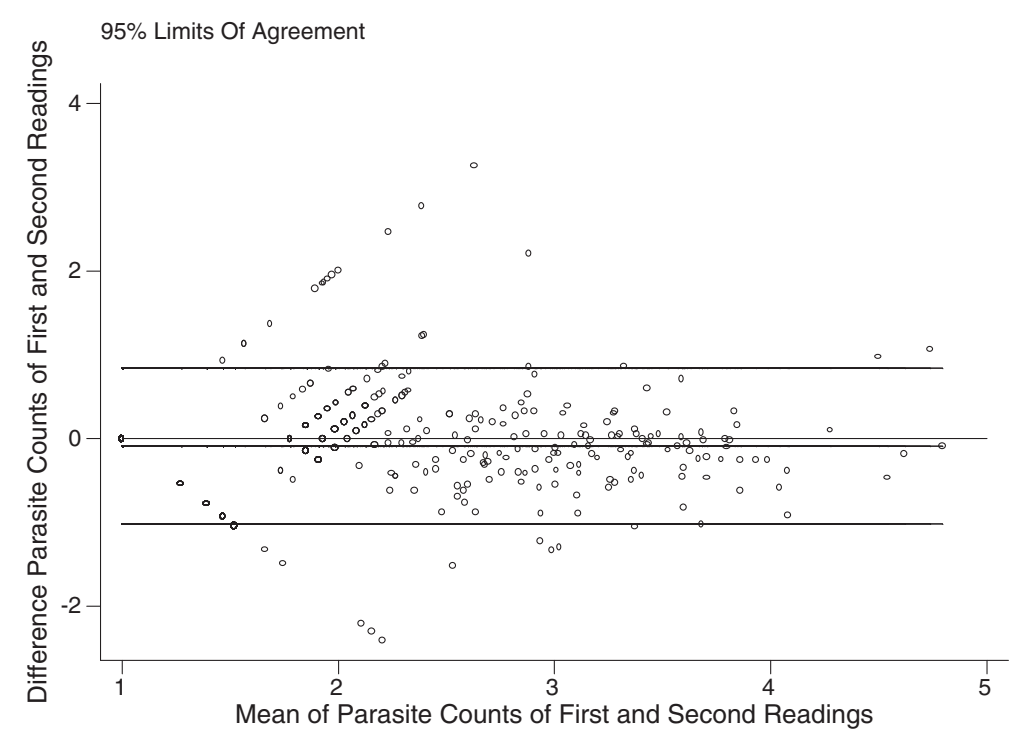

Figure 2 Difference against average of transformed parasite counts of first and second readings, with $95 \%$ limits of agreement using all data (932 slides).

Parasite density estimation is highly valuable for the clinician, as it is an important determinant of treatment schedules for $P$. falciparum. If parasite density exceeds $10 \%$ in P. falciparum, exchange transfusion may be indicated $[14,15]$. A variety of studies have clearly demonstrated that microscopic diagnosis of malaria can vary greatly in its accuracy, particularly at low parasitaemia rates $[12,16,17]$. This variation in specificity and sensitivity is routinely observed in clinical settings, where a high proportion of reporting patients are parasitaemic and parasite densities are relatively high. In this study, the weighted kappa statistic, widely used as a chancecorrected measure for nominal agreement, showed excellent inter-observer agreement $\left(\kappa_{\mathrm{w}}=0.7926 ; 95 \%\right.$ CI [0.7588, $0.8263] ; p=0.01$ ). Inter-observer agreement for slides read as negative by one observer, or as containing more than 100 parasites per $\mu \mathrm{l}$ was excellent: 97\% (493/506) and 92\% (145/158), respectively. Dowling and Shute compared parasite counts obtained by examination of thin and thick smears and conducted that parasite losses of 60 to $90 \%$ occurred with thick films, whereas since thin films are fixed after drying and before staining, they assumed no significant loss of parasites during staining [18].

In a series of parasite dilutions, studies have found that thick films tended to measure parasite densities around one log lower than the number calculated to be in the dilution and this did not vary by microscopist $[13,19]$. O'Meara et al. [19] have shown that parasitaemia from the thick smear averaged 10\% lower than the total mean $(p=0.001)$ and they have also shown that white blood cells were much less uniformly distributed that the parasites. They also confirmed that up to $60 \%$ of parasites were obscured in the thick film or lost during the process of red cell lyses and parasite staining. In this study, agreement was compared between two highly qualified microscopists according to parasite densities.

In contrast, the inter-observer agreement for slides read by one observer as containing $1-100$ parasites/ $\mu \mathrm{l}$ was poor, 36\% (96/268). The concordance correlation co-efficient [5] was 0.835 , 95\% CI (0.816-0.855) using logarithm-transformed parasite counts, and yielded a regression line with near-perfect concordance between the first and the second readings: an average difference of $-0.088 \pm 0.474$ [10] [Figure 2]. Greenwood and Armstrong [20] have suggested that variation in parasite density depends in variability in the volume of blood used to prepare thick films being less than the variability in white blood cell count in the population they studied.

When two parasite counts for the same slide were compared, Killian et al. found considerable variability, with one reading being 0.12 to ten times the other [21]. They examined inter-rater variability in the results of malaria microscopy in epidemiological studies using 711 thick blood films re-read by four experienced microscopists. They also calculated parasite density by counting the number of trophozoites in 100 oil immersion fields and multiplying by four to give parasites per microlitre, assuming a blood volume of approximately $0.25 \mu \mathrm{l}$ per 100 microscope fields. There was significantly less variability at parasite densities above $500 / \mu l, 0.2$ to 3.6 times. Overall, for variation between readers, O'Meara et al. stated that discrepancies in parasite densities reported by experienced clinic microscopists decreased with increasing mean density and trends were similar for $P$. falciparum and for $P$. vivax when they were considered separately [22]. When agreement between readers 
is required, it is important to apply an identical technique which seems to be more important than increasing the number of microscope fields read [22]. In another study, these authors found a significant inverse correlation between discrepancy among microscopists and mean parasite density [23]. Furthermore, they suggested that random chance in the selection of fields to examine may play a large part in reader discrepancy, especially with low parasitaemia. In a recent review, Makler et al. concluded that factors such as undertraining of microscopists, lack of microscopes and staining materials, and processing and reading large numbers of blood smears, dramatically increased the range for error [24]. Using the method described by Alexander et al. [25], similar findings were observed in the present study (see Additional file 1). When back-transformed to the original count, the limits with agreements increase with parasite density, and are much wider.

Since most elimination efforts will need to deal with both low parasitaemia and non-falciparum species, diagnosis becomes a major challenge for elimination programmes. Bowers et al. have shown differences between methods using the same microscopy staff, but reader technique itself clearly contributes to the accuracy of parasitaemia estimates [26]. Although the propensity of a gametocyte carrier to transmit infection is related to the density of gametocytaemia, individuals with very low gametocyte numbers can still transmit malaria infection and can be an important part of the reservoir of infection. Thus, elimination programmes will need to detect and treat all potential transmitters of infection with a more sensitive detection test. The slide readers in this study were all experienced malaria microscopists and the results may be different with less experienced readers. In the light of this and under low parasite prevalence, low parasite rates, and inadequate equipment conditions, for any parasite density less than 100 parasites/ $\mu \mathrm{l}$ at least two experienced microscopists should blind read the slide.

\section{Conclusion}

Improved means to detect asymptomatic persons with low parasitaemia will be crucial to malaria elimination. These results suggest a high reproducibility for slides reported as negative or as having more than 100 parasites per $\mu \mathrm{l}$. However, low parasitaemia $(<100$ parasites/ $\mu$ l are less reproducible and should be re-examined carefully. In addition, a uniform counting protocol should be used and the number of white blood cells counted should be increased in order to improve inter-reader agreement. Until rapid, reproducible and quantitative PCR for malaria is widely available at low cost, microscopy will remain the method of choice for parasite density determination in malaria elimination phase as most African countries are observing a decrease in malaria prevalence.

\section{Additional file}

\begin{abstract}
Additional file 1: Between-readers variation in asexual parasites counts. Title: Variation between readers in asexual parasites counts using Alexander et al. (2010) methods. Description: When back-transformed to the original parasite density count, the limits with agreements increase with parasite density, and are much wider.
\end{abstract}

\section{Competing interests}

The authors have declared that they have no competing interests.

\section{Authors' contributions}

$A D$ and MD were the reference microscopists; OKD was the trainer in malaria slide microscopy and served as the third reference microscopist for the entire Mali-Tulane TMRC grant. AD, MD and OKD participated in drafting the manuscript and supervised the collection of samples. MAB, MD, ESJ, JCR, OKD and DJK conceived the study and participated in its design. OKD and MAB supervised all aspects of the study carried out in Bancoumana and the drafting of the manuscript. MAB, JCR and ESJ performed all the statistical analysis for the study. All authors read and approved the final manuscript.

\section{Acknowledgements}

The authors are grateful to the population of Bancoumana and the TMRCMRTC-DEAP field and laboratory teams for the quality of data collection on the field sites at Bancoumana, and to the Dean Pr Issa Traoré of the Ecole Nationale de Médecine et de Pharmacie for his full support of the MaliTulane TMRC activities. The project was funded by the Mali-Tulane Tropical Research Centre, funded by the NIH-extramural research programme (NIAID P50 Al 39469) MAB's PhD activities in Mali were supported by the WHO/TDR through the Special Programme for Research and Training in Tropical Diseases, grant ID-930736.

Received: 3 May 2013 Accepted: 15 September 2013

Published: 22 September 2013

\section{References}

1. Kricker A, Armstrong BK, English DR: Sun exposure and non-melanocytic skin cancer. Cancer Causes Control 1994, 5:367-392.

2. Payne D: Use and limitations of light microscopy for diagnosing malaria at the primary health care level. Bull World Health Organ 1988, 66:621-626.

3. Dolo A, Camara F, Poudiougo B, Toure A, Kouriba B, Bagayogo M, Sangare D, Diallo M, Bosman A, Modiano D, et al: Epidemiology of malaria in a village of Sudanese savannah area in Mali (Bancoumana) 2. Entomoparasitological and clinical study. Bull Soc Pathol Exot 2003, 96:308-312.

4. Cohen J: Weighted kappa: nominal scale agreement with provision for scaled disagreement or partial credit. Psychol Bull 1968, 70:213-220.

5. Lin LI: A concordance correlation coefficient to evaluate reproducibility. Biometrics 1989, 45:255-268.

6. Bland JM, Altman DG: Statistical methods for assessing agreement between two methods of clinical measurement. Lancet 1986, 1:307-310.

7. Bland JM, Altman DG: Comparing methods of measurement: why plotting difference against standard method is misleading. Lancet 1995, 346:1085-1087.

8. Maclure M, Willett WC: Misinterpretation and misuse of the kappa statistic. Am J Epidemiol 1987, 126:161-169.

9. Voller A, Draper CC: Immunodiagnosis and sero-epidemiology of malaria. Br Med Bull 1982, 38:173-177

10. Santana-Morales MA, Afonso-Lehmann RN, Quispe MA, Reyes F, Berzosa P, Benito A, Valladares B, Martinez-Carretero E: Microscopy and molecular biology for the diagnosis and evaluation of malaria in a hospital in a rural area of Ethiopia. Malaria J 2012, 11:199.

11. Warhurst DC, Williams JE: ACP Broadsheet no 148. July 1996. Laboratory diagnosis of malaria. J Clin Pathol 1996, 49:533-538.

12. Bejon P, Andrews L, Hunt-Cooke A, Sanderson F, Gilbert SC, Hill AV: Thick blood film examination for Plasmodium falciparum malaria has reduced sensitivity and underestimates parasite density. Malaria J 2006, 5:104. 
13. Shelat SG, Lott JP, Braga MS: Considerations on the use of adjunct red blood cell exchange transfusion in the treatment of severe Plasmodium falciparum malaria. Transfusion 2010, 50:875-880.

14. Mordmuller B, Kremsner PG: Hyperparasitemia and blood exchange transfusion for treatment of children with falciparum malaria. Clin Infect Dis 1998, 26:850-852.

15. Craig MH, Sharp BL: Comparative evaluation of four techniques for the diagnosis of Plasmodium falciparum infections. Trans $R$ Soc Trop Med Hyg 1997, 91:279-282.

16. Tham JM, Lee SH, Tan TM, Ting RC, Kara UA: Detection and species determination of malaria parasites by PCR: comparison with microscopy and with ParaSight-F and ICT malaria Pf tests in a clinical environment. J Clin Microbiol 1999, 37:1269-1273.

17. Dowling MA, Shute GT: A comparative study of thick and thin blood films in the diagnosis of scanty malaria parasitaemia. Bull World Health Organ 1966, 34:249-267.

18. Mya MM, Saxena RK, Bhakat P, Roy A: Effect of serum dilution in diagnosis of malaria in community. J Commun Dis 2000, 32:28-32.

19. O'Meara WP, Barcus M, Wongsrichanalai C, Muth S, Maguire JD, Jordan RG, Prescott WR, McKenzie FE: Reader technique as a source of variability in determining malaria parasite density by microscopy. Malaria J 2006, 5:118.

20. Greenwood BM, Armstrong JR: Comparison of two simple methods for determining malaria parasite density. Trans R Soc Trop Med Hyg 1991, 85:186-188

21. Kilian AH, Metzger WG, Mutschelknauss EJ, Kabagambe G, Langi P, Korte R, von Sonnenburg F: Reliability of malaria microscopy in epidemiological studies: results of quality control. Trop Med Int Health 2000, 5:3-8.

22. O'Meara WP, McKenzie FE, Magill AJ, Forney JR, Permpanich B, Lucas C, Gasser RA Jr, Wongsrichanalai C: Sources of variability in determining malaria parasite density by microscopy. Am J Trop Med Hyg 2005, 73:593-598.

23. Bland JM, Altman DJ: Regression analysis. Lancet 1986, 1:908-909.

24. Makler MT, Palmer CJ, Ager AL: A review of practical techniques for the diagnosis of malaria. Ann Trop Med Parasitol 1998, 92:419-433.

25. Alexander N, Schellenberg D, Ngasala B, Petzold M, Drakeley C, Sutherland C: Assessing agreement between malaria slide density readings. Malaria J 2010, 9:4

26. Bowers KM, Bell D, Chiodini PL, Barnwell J, Incardona S, Yen S, Luchavez J, Watt H: Inter-rater reliability of malaria parasite counts and comparison of methods. Malaria J 2009, 8:267.

doi:10.1186/1475-2875-12-335

Cite this article as: Billo et al.: Inter-observer agreement according to malaria parasite density. Malaria Journal 2013 12:335.

\section{Submit your next manuscript to BioMed Central and take full advantage of:}

- Convenient online submission

- Thorough peer review

- No space constraints or color figure charges

- Immediate publication on acceptance

- Inclusion in PubMed, CAS, Scopus and Google Scholar

- Research which is freely available for redistribution 